archives

of thermodynamics

Vol. 36(2015), No. 1, 3-18

DOI: $10.1515 /$ aoter-2015-0001

\title{
Thermal analysis of heat and power plant with high temperature reactor and intermediate steam cycle
}

\author{
ADAM FIC* \\ JAN SKŁADZIEŃ \\ MICHAŁ GABRIEL
}

Silesian University of Technology, Institute of Thermal Technology, Konarskiego 22, 44-100 Gliwice, Poland

\begin{abstract}
Thermal analysis of a heat and power plant with a high temperature gas cooled nuclear reactor is presented. The main aim of the considered system is to supply a technological process with the heat at suitably high temperature level. The considered unit is also used to produce electricity. The high temperature helium cooled nuclear reactor is the primary heat source in the system, which consists of: the reactor cooling cycle, the steam cycle and the gas heat pump cycle. Helium used as a carrier in the first cycle (classic Brayton cycle), which includes the reactor, delivers heat in a steam generator to produce superheated steam with required parameters of the intermediate cycle. The intermediate cycle is provided to transport energy from the reactor installation to the process installation requiring a high temperature heat. The distance between reactor and the process installation is assumed short and negligable, or alternatively equal to $1 \mathrm{~km}$ in the analysis. The system is also equipped with a high temperature argon heat pump to obtain the temperature level of a heat carrier required by a high temperature process. Thus, the steam of the intermediate cycle supplies a lower heat exchanger of the heat pump, a process heat exchanger at the medium temperature level and a classical steam turbine system (Rankine cycle). The main purpose of the research was to evaluate the effectiveness of the system considered and to assess whether such a three cycle cogeneration system is reasonable. Multivariant calculations have been carried out employing the developed mathematical model. The results have been presented in a form of the energy efficiency and exergy efficiency of the system as a function of the temperature drop in the high temperature process heat exchanger and the reactor pressure.
\end{abstract}

${ }^{*}$ Corresponding Author. E-mail: Adam.Fic@polsl.pl 
Keywords: Cogeneration system; High temperature reactor; Exergy analysis; Gas heat pump; Numerical simulation

\section{Introduction}

Nuclear reactors operating in the energy sector are presently used mainly for electricity production. However, nuclear reactors are also an attractive alternative for the use in cogeneration systems both for electricity and heat production. Cogeneration systems with nuclear reactors are effective not only from energy saving and economical point of view, but they are also environmentally friendly, as they do not emit carbon dioxide. Other possible solutions of systems used to decrease this emission are presented, e.g., in $[1,2]$. The high temperature reactor (HTR) [3] seems to be one of the most promising technologies in this context. Firstly, these types of reactors are regarded as one of the safest nuclear technologies due to very strong negative temperature reactivity effects, which self-stabilize the reactor operation. Moreover, the reactor fuel is covered by cladding, which is resistant to very high temperatures, even those appearing in accidental conditions. On the other hand, helium used in HTRs as a reactor coolant can achieve temperatures at the level of 1000-1250 K. This means that the heat from these reactors can be also used to supply industrial processes requiring heat from sources of such high temperature levels. Hydrogen or ammonia production and coal gasification are the examples of such processes. The temperature levels of order $1250 \mathrm{~K}$ can be achieved in foreseeable generation IV very high temperature reactors (VHTRs) rather in the far future. According to current opinion, in the first installed HTRs, the outlet temperature will rather not be higher than about $1020 \mathrm{~K}$. Such temperature is too low for some high temperature processes. Additionally, there are no regulations stating the required distance between the reactor and industrial installations. A necessary distance of the order of $1-2 \mathrm{~km}$ is very probable. A possible solution considered recently to satisfy the above requirements is to use steam with parameters commonly used in coal power plants (about $16 \mathrm{MPa}, 810 \mathrm{~K}$ ) as a heat carrier employed to transport energy from the reactor to the industrial system. As a result, the energy carrier delivering the heat to a high temperature process has to be heated up in this system to the desired temperature. This can be accomplished electrically or by using a heat pump in an additional cycle of the system. This kind of the three cycle cogeneration system with a HTR and a gaseous heat pump is 
analysed in the paper.

Helium used as the reactor coolant is also employed to produce superheated steam of the intermediate cycle in a steam generator and to accomplish the Brayton cycle. As mentioned before, this steam, as the heat carrier of the intermediate cycle, transports energy from the reactor installation to the process installation. The third gaseous (argon) heat pump cycle is provided in the system to obtain the highest temperature level of a heat carrier required by the process. Steam from the intermediate cycle in the system delivers heat to the evaporator of the heat pump, and next is used as a working carrier in the classical steam turbine system. Topics of the cogeneration systems considered depend strongly on the desired temperatures in the process heat exchangers. The temperature levels for technological heat may be different and they are in the range from below $370 \mathrm{~K}$ up to 1270 K [3]. In Poland, the processes of hydrogen production and hard coal gasification are especially interesting and the demand for temperature level is in the range 390-1170 $\mathrm{K} \mathrm{[4]}$. The other possibilities of heat utilization from the reactors are presented in [4-6]. The application of nuclear reactor installation supplying technological heat and working with gas compressor heat pump is considered in $[4,7]$ together with some variants of such systems. In the system considered in this paper, two temperature levels of technological heat have been assumed, i.e., about $1070 \mathrm{~K}$ and $670 \mathrm{~K}$, as in the process of a sulphur-iodine cycle of hydrogen production [8].

The main purpose of the paper is to evaluate the effectiveness of the considered three cycle cogeneration system consisting of a HTR reactor and a high temperature heat pump (HP). An assessment of whether this system is reasonable if the distance between the reactor and the process installations is of the order of 1-2 km, is also an important aim of the work. For this purpose, multivariant calculations of the system have been carried out employing worked out mathematical model. The model has been implemented at the platform of the commercial code EBSILON [9]. The main results presented in the paper have a form of the energy efficiency and exergy efficiency of the system as a function of the temperature drop in the high temperature process heat exchanger and the reactor pressure for various temperature differences between carriers in the regenerative heat exchanger of the heat pump, for the different helium temperature at the reactor outlet, assuming that the reactor is located very close or $1 \mathrm{~km}$ from the process installation. Actually, to the best knowledge of the authors, there are no published results of such kind of investigations. 


\section{Cogeneration system with high temperature nuclear reactor}

The considered basic system is presented in Fig. 1a. As mentioned previously, it consists of three loops: the reactor helium coolant loop, the steam intermediate loop and the argon HP loop. Reactor 1 in the considered system heats up helium, which is next expanding in helium turbine 2. In steam generator 3 , the heat is transferred to water of the intermediate cycle. Next, helium is cooled down in the exchanger 4 and compressed in compressor 5 . Turbine 2 has a common shaft with compressor 5 and the first electric generator. Feed water of pressure equal to $15 \mathrm{MPa}$ flowing to the steam generator 3 is heated up, evaporated and the obtained steam is superheated up to $813 \mathrm{~K}$ in the considered analysis. This steam is used to transport energy to heat exchanger 6 and next to heat exchanger 7 . Heat exchanger 6 is the lower heat source for the gas compression HP, and heat exchanger 7 is a source of the technological heat at the medium temperature level. The main stream of the steam from exchanger 7 flows to steam turbine system, which has three parts: high- 8 , medium- 10, and low-pressure 11. All turbines have a common shaft with the second electric generator. A small amount of steam from exchanger 7 is used in superheater 9 to overheat the steam between turbine 8 and turbine 10. Outlet steam from turbine 11 condenses in condenser 12, which is cooled by water supplied from pump 13 . The condensate from separators, superheater 9, and regenerative preheater 16, as well as small amount of steam taken before superheater 9 are directed to the degasifier 17. Feed water from degasifier 17, after compressing in feed water pump 18, is heated in regenerative preheater 15 by steam taken before the superheater. Next, the feed water flows to steam generator 3. Gas compressor heat pump cycle consists of heat exchanger 6 being a lower heat source for the heat pump system, regenerative exchanger 20, compressor 21, high temperature heat exchanger 22 being an upper heat source of the HP, and turbine 19. Moreover, a system with three stage compression in the HP cycle has been considered in the analysis. The system with multistage compression in the HP cycle can produce higher heat flux transferred to the high temperature process than in the case of one stage compression with the same flux of a heat carrier. The scheme of the system with three stage compression in the HP is presented in Fig. 1b. It is worth mentioning that in the case of three stage compression in the argon HP cycle, the ratio of pressure in the first compressor 21 is greater than in the next ones. These pressure 
a)

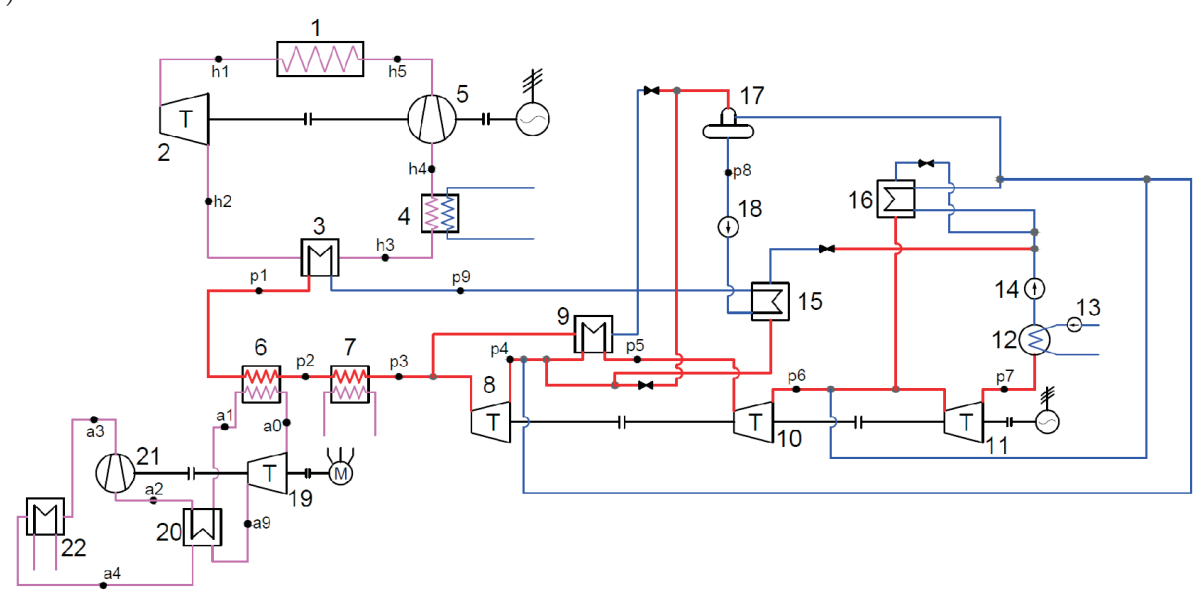

b)

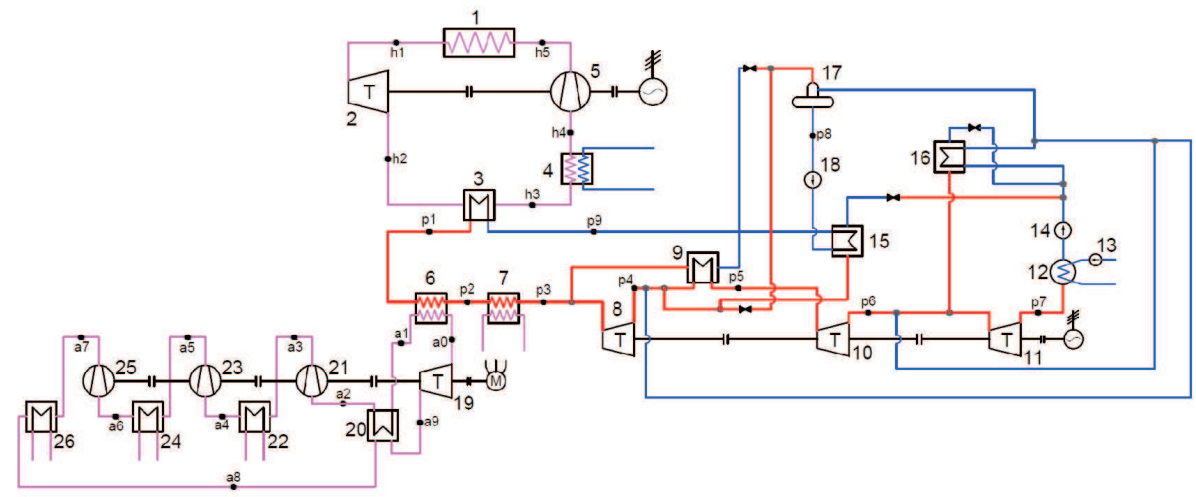

Figure 1: Schemes of the considered three loop cogeneration systems with HTR containing helium cooling loop, steam intermediate loop and argon HP loop; 1a basic system with one stage compression in HP, $1 \mathrm{~b}$ - system with three stage compression; 1 - HTR reactor, 2 - helium turbine, 3 - steam generator, 4 cooler, 5 - helium compressor, 6 - steam-argon exchanger, 7 - medium process heat exchanger, 8 - high pressure turbine, 9 - superheater, 10 - medium pressure turbine, 11 - low pressure turbine, 12 - condenser, 13 - water pump, 14 condenser pump, 15 - high pressure regenerative preheater, 16 - low pressure regenerative preheater, 17 - degasifier, 18 - feed pump, 19 - argon turbine, 20 - regenerative heat exchanger, 21, 23,25 - compressors, 22, 24, 26 - sections of high temperature process heat exchanger.

ratios are determined by the assumed maximal and minimal temperatures in the upper HP exchanger (or exchangers in the cases of multistage com- 
pression). Generally, cogeneration systems with HTRs may also be used for supplying heat for heating systems using additional streams of steam for such purposes from, e.g., bleedings between neighbouring parts of the steam turbine. Such possibility was however not included in the analysis.

\section{Results of numerical calculations and conclusions}

The numerical calculations have been performed using the EBSILON code [9]. Steam in the simulations was treated as a real gas, while helium and argon were assumed to be ideal gases. The mathematical model of the considered system consists of mass and energy balance equations, working fluid state equations and equations of characteristic processes. The processes in compressors, turbines and pumps were assumed to be adiabatic and nonreversible ones.

As it was mentioned before, steam of the prescribed pressure equal to $15 \mathrm{MPa}$ and temperature $813 \mathrm{~K}$ is produced in the steam generator 3. Minimal temperature difference between heat carriers in the exchangers 3 and 6 was assumed equal to $25 \mathrm{~K}$. Isentropic efficiency of the gas turbines was equal to 0.9 , steam turbines 0.88 , compressors 0.86 , mechanical efficiencies of turbines, $\eta_{m T}$, and compressors, $\eta_{m c}$, were 0.99, pumps $\eta_{p}-0.95$, and generators $\eta_{g}-0.99$. A series of simulations have been carried out for the presented systems for various temperature drop, $d T_{p r}$, in the high temperature process heat exchanger (or exchangers in the case of multistage compression), various minimal temperature in this exchanger, $T_{p r \text { min }}$, various pressure, $p_{r}$, at the reactor inlet assuming that the reactor is located close to technological installation (i.e., neglecting the drop of the temperature and pressure in the pipes of the intermediate circuit) and situated $1 \mathrm{~km}$ from this installation.

The drop of the heat and pressure in the pipe with the steam and feed water of the intermediate circuit was estimated numerically using commercial Engineering Equation Solver (EES) software package [13], which employs REFPROP library [14] for the steam parameters. Colebrooke's formula [10] was applied to determine the flow resistance coefficient. Calculations have been worked out by dividing pipes into many short segments. The mentioned drops occurred negligible in the feed water pipe. The estimated pressure drop in the $1 \mathrm{~km}$ steam pipe was equal to $2.3 \mathrm{MPa}$, and the temperature drop $10 \mathrm{~K}$, when the assumed steam velocity was $50 \mathrm{~m} / \mathrm{s}$. 
For instance, in the case of the reactor pressure $p_{r}=7 \mathrm{MPa}$, reactor outlet temperature $T_{\text {rout }}=1023 \mathrm{~K}$, minimal temperature in the high temperature process heat exchanger $T_{\text {pr min }}=1073 \mathrm{~K}$, temperature drop in the high temperature process heat exchanger $d T_{p r}=100 \mathrm{~K}$, the following results have been obtained:

- reactor thermal power: $\dot{Q}_{1}=299.0 \mathrm{MW}$,

- rate of heat in the steam generator: $\dot{Q}_{3}=268.4 \mathrm{MW}$,

- rate of heat in the process heat exchanger 6: $\dot{Q}_{6}=37.0 \mathrm{MW}$,

- rate of heat in the high temperature process heat exchanger 22: $\dot{Q}_{22}=$ 62.6 MW,

- internal power of the helium turbine: $N_{T 2}=168.2 \mathrm{MW}$,

- internal power of the helium compressor: $N_{c 2}=144.6 \mathrm{MW}$,

- internal power of the high pressure steam turbine: $N_{T 8}=10.9 \mathrm{MW}$,

- internal power of the medium pressure steam turbine: $N_{T 10}=19.0 \mathrm{MW}$,

- internal power of the low pressure steam turbine: $N_{T 11}=47.5 \mathrm{MW}$,

- internal power of the argon turbine: $N_{T 19}=43.0 \mathrm{MW}$,

- internal power of the argon compressor: $N_{c 21}=78.0 \mathrm{MW}$.

Calculated pressure and temperature at selected nodes of the system (Fig. 1) were:

$p_{h 1}=6.9 \mathrm{MPa}, T_{h 1}=1023 \mathrm{~K}, p_{h 2}=3.8 \mathrm{MPa}, T_{h 2}=833 \mathrm{~K}, T_{h 3}=528 \mathrm{~K}$, $p_{h 5}=7.0 \mathrm{MPa}, T_{h 5}=683 \mathrm{~K}$,

$p_{p 1}=15.0 \mathrm{MPa}, T_{p 1}=813 \mathrm{~K}, T_{p 2}=720 \mathrm{~K}, p_{p 3}=14.9 \mathrm{MPa}, T_{p 3}=633 \mathrm{~K}$, $p_{p 4}=6.9 \mathrm{MPa}, T_{p 4}=558 \mathrm{~K}, p_{p 7}=0.0056 \mathrm{MPa}, T_{p 7}=308 \mathrm{~K}, T_{p 9}=446 \mathrm{~K}$, $p_{a 0}=0.100 \mathrm{MPa}, T_{a 0}=754 \mathrm{~K}, T_{a 1}=798 \mathrm{~K}, T_{a 2}=1048 \mathrm{~K}, p_{a 3}=$ $0.128 \mathrm{MPa}, T_{a 3}=1173 \mathrm{~K}, T_{a 4}=1073 \mathrm{~K}, T_{a 9}=823 \mathrm{~K}$, where the indices denote points of the system shown in Fig. 1.

For the considered cases, the overall thermal efficiency and exergy efficiency were analysed. The overall thermal efficiency $\eta_{E}$ was defined for the system as

$$
\begin{aligned}
\eta_{E}= & \frac{\dot{Q}_{7}+\dot{Q}_{22}+\eta_{g}\left[\eta_{m T s} \sum N_{T s}+\eta_{m T A r} N_{T A r}-\frac{1}{\eta_{m c A r}} \sum N_{c A r}\right]}{\dot{Q}_{1}}+ \\
& +\frac{\eta_{g}\left(\eta_{m T H e} N_{T H e}-\frac{1}{\eta_{m c H e}} N_{c H e}\right)-\frac{1}{\eta_{p} \sum N_{p}}}{\dot{Q}_{1}}
\end{aligned}
$$


and the exergy efficiency, $\eta_{B}$, as

$$
\begin{aligned}
\eta_{B}= & \frac{\dot{Q}_{7}\left(1-\frac{293}{T_{7}}\right)+\dot{Q}_{22}\left(1-\frac{293}{T_{22}}\right)+\eta_{g}\left[\eta_{m T s} \sum N_{T s}+\eta_{m T A r} N_{T A r}-\frac{1}{\eta_{m c A r}} \sum N_{c A r}\right]}{\dot{Q}_{1}\left(1-\frac{293}{T_{1}}\right)}+ \\
& +\frac{\eta_{g}\left(\eta_{m T H e} N_{T H e}-\frac{1}{\eta_{m c H e}} N_{c H e}\right)-\frac{1}{\eta_{p}} \sum N_{p}}{\dot{Q}_{1}\left(1-\frac{293}{T_{1}}\right)}
\end{aligned}
$$

where sums are extended over all turbines (index $T$ ), compressors $(c)$ and pumps $(p)$, and indexes $H e, s$, and $A r$ relate respectively to helium, steam and argon. The temperatures $T_{7}$ and $T_{22}$ denote temperatures of the isothermal processes, which are supplied by the heat delivered from the process heat exchangers 7 and 22 and assumed $15 \mathrm{~K}$ lower than the minimal temperature of the energy carrier in the proper exchanger. In the case of the reactor, the temperature $T_{1}$ was assumed as the mean thermodynamic temperature of helium in the reactor, i.e.,

$$
T_{1}=\frac{\Delta h_{1}}{\Delta s_{1}},
$$

where $\Delta h_{1}$ and $\Delta s_{1}$ denote the increase of specific enthalpy and entropy, respectively, of helium in the reactor.

Selected graphs of exergy and overall thermal efficiency of the considered systems are presented in Figs. 2-6. In the considered base case, the minimal temperature in the high temperature process heat exchanger, $T_{p r m i n}$, was $1073 \mathrm{~K}$, and the pressure, $p_{r}$, at the reactor inlet $6.9 \mathrm{MPa}$, the reactor was located close to technological installation and one stage compression was assumed in the HP. The results for this case are presented in Fig. 2.

The obtained exergy efficiency is higher than energy efficiency by about ten percent. The level of exergy efficiency results from its definition [11]. Another approach is presented in [12]. It is based on the assumed exergy of a nuclear fuel, which is very controvertible, and leads to small values of exergy efficiency for nuclear power plants. 

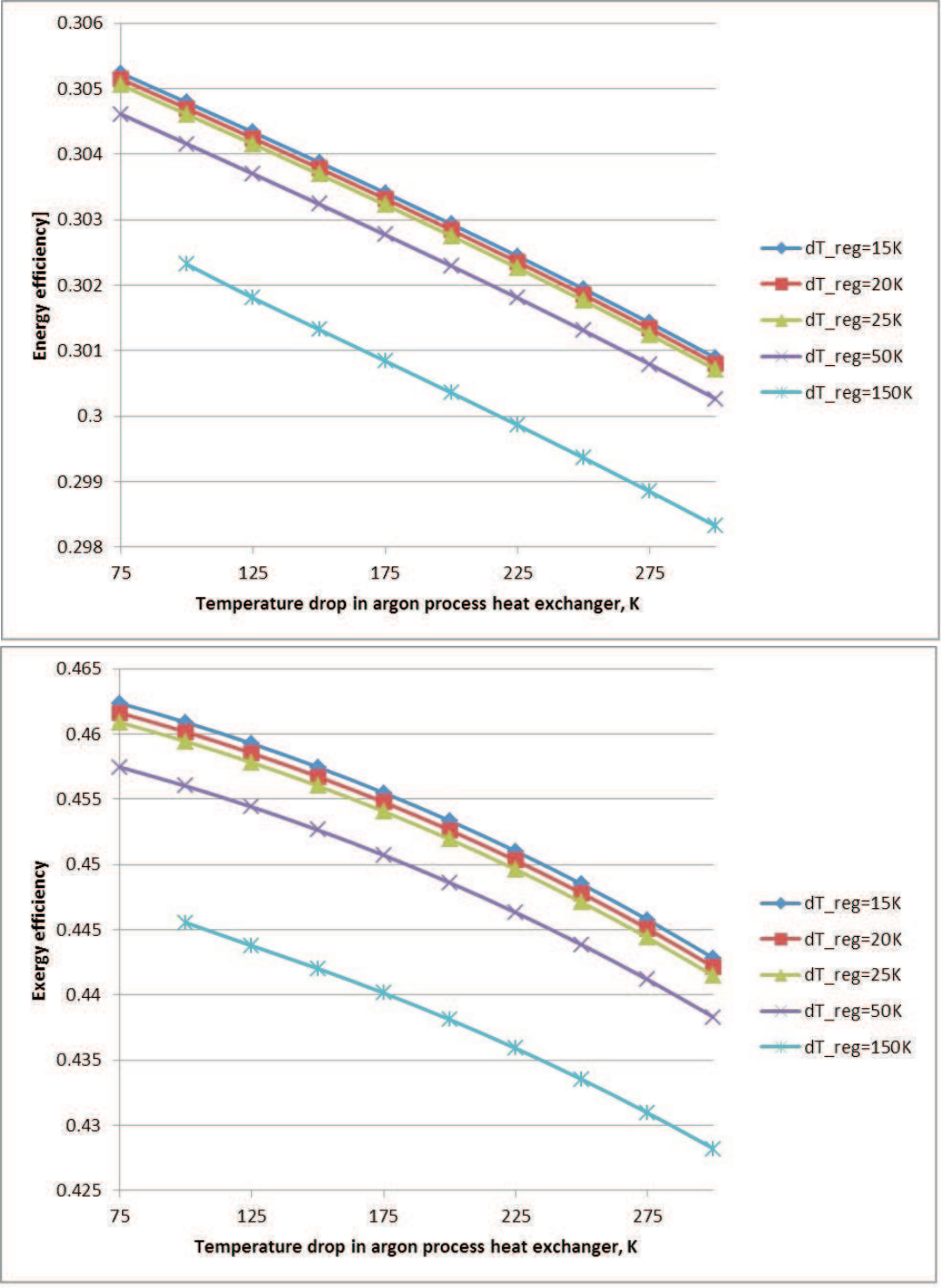

Figure 2: Overall thermal energy and exergy efficiency of the system with one stage of the argon compressor versus argon temperature drop in the high temperature exchanger 22 for various temperature differences in the argon regenerative heat exchanger 20, for reactor outlet helium parameters $1023 \mathrm{~K}, 6.9 \mathrm{MPa}$, and for minimum temperature in high temperature process heat exchanger 3 equal to $1073 \mathrm{~K}$; reactor is located close to technological process and steam turbine system. 

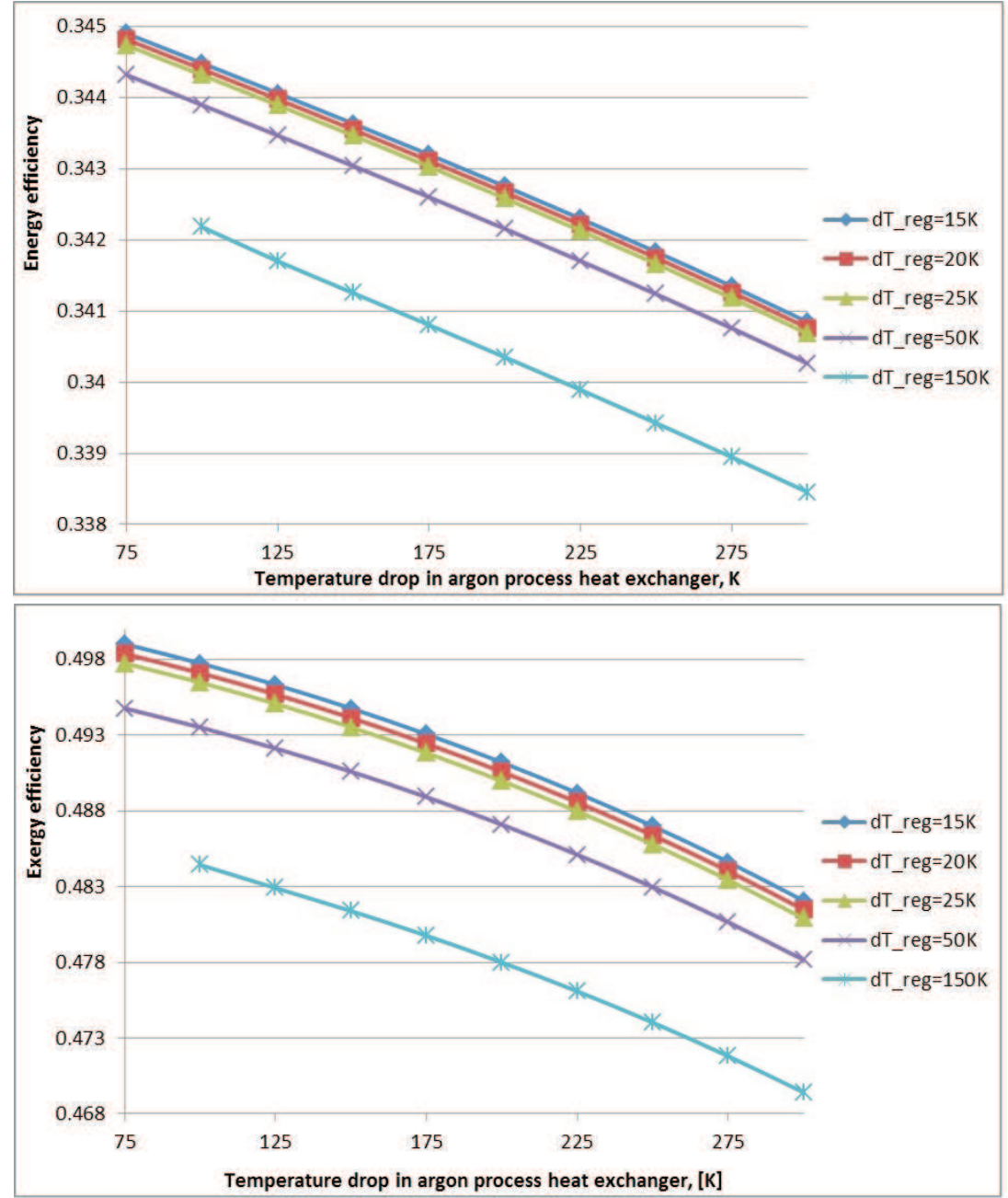

Figure 3: Overall thermal energy and exergy efficiency of the system with one stage of the argon compressor versus argon temperature drop in high temperature exchanger 22 for various temperature differences in the argon regenerative heat exchanger 20, for reactor outlet helium parameters $1023 \mathrm{~K}, 6.9 \mathrm{MPa}$. 

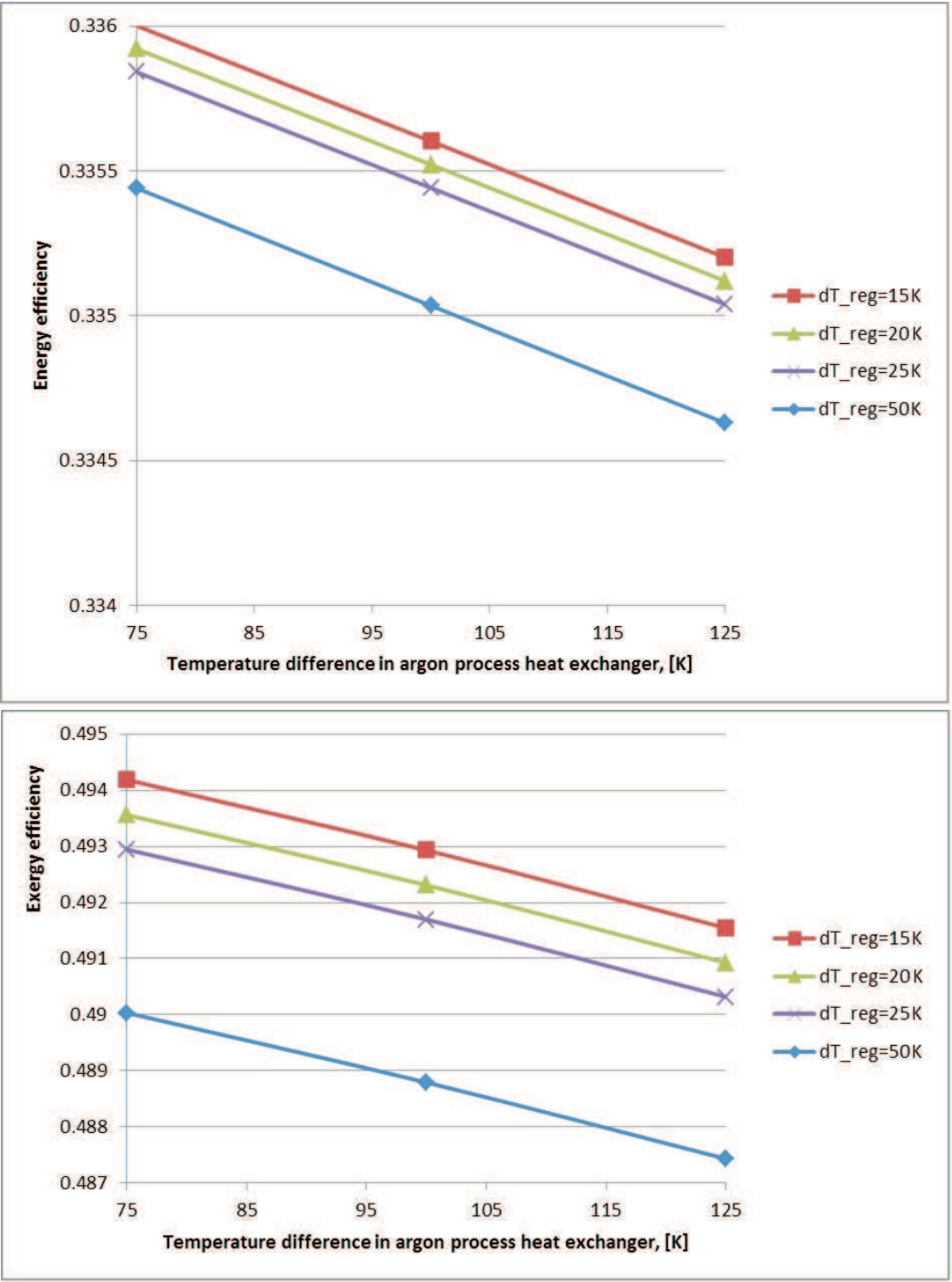

Figure 4: Overall thermal energy and exergy efficiency of the system with one stage of the argon compressor versus argon temperature drop in the high temperature heat exchanger 22 for various temperature difference in the argon regenerative heat exchanger 20, for reactor outlet helium parameters $1123 \mathrm{~K}, 6.9 \mathrm{MPa}$, when the reactor is located $1 \mathrm{~km}$ from technological process and steam turbine system. 

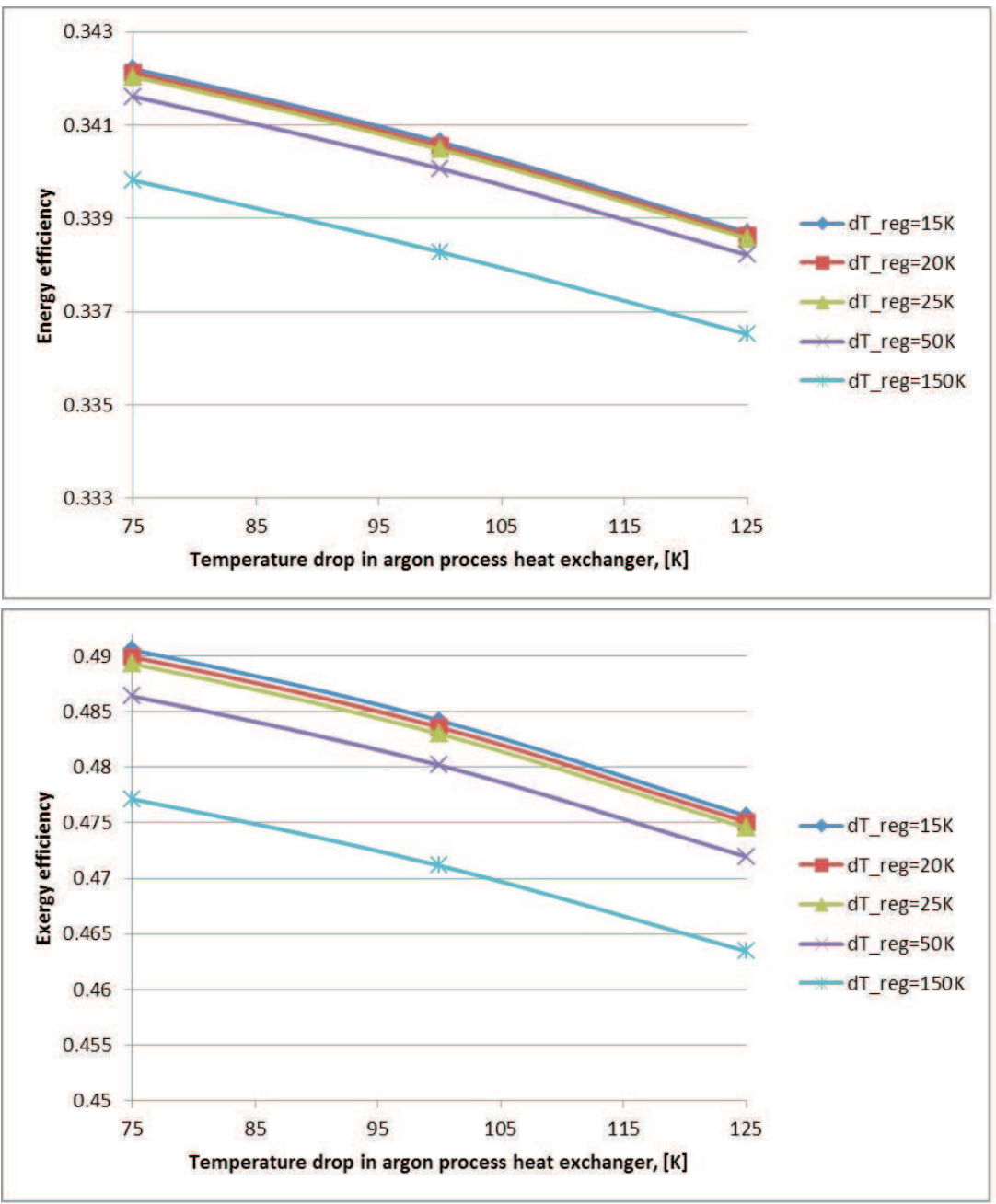

Figure 5: Overall thermal energy and exergy efficiency of the system with three stage of the argon compression versus argon temperature drop in the high temperature exchanger 22 for various temperature differences in the argon regenerative heat exchanger 20, for reactor outlet helium parameters $1123 \mathrm{~K}, 6.9 \mathrm{MPa}$. 

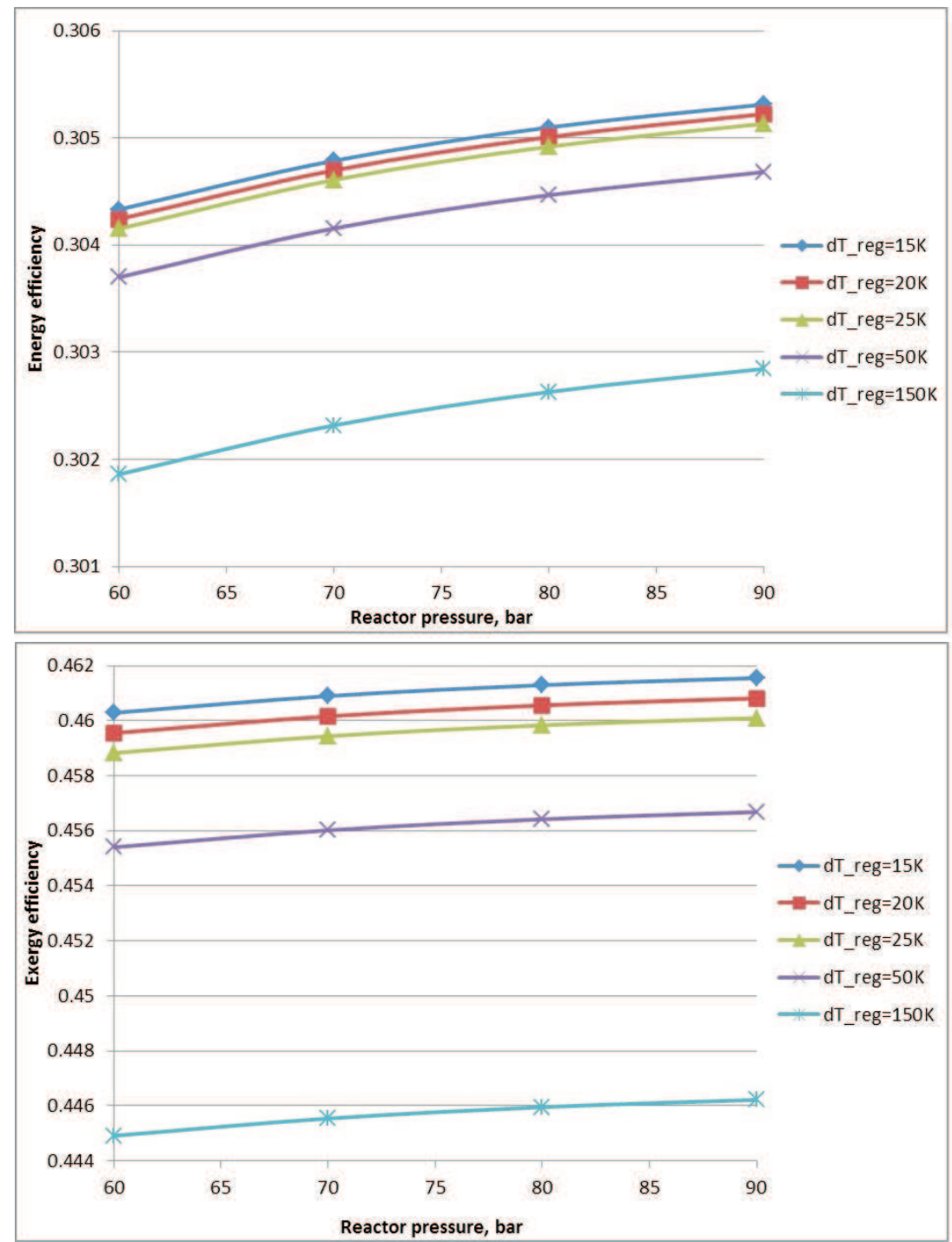

Figure 6: Overall thermal energy and exergy efficiency of the system with one stage of the argon compression versus the reactor pressure for various temperature differences in the argon regenerative heat exchanger 20. 
Figures 2 and 3 show the exergy efficiency and the overall thermal efficiency of the system with one stage compression in the HP versus temperature decrease $d T_{p r}$ in the high temperature process heat exchanger for various temperature differences $\mathrm{dT}$ _reg in the regenerator of the HP unit, the reactor outlet pressure $p_{r}=6.9 \mathrm{MPa}$, minimum temperature in the high temperature process heat exchanger $T_{p r \min }=1073 \mathrm{~K}$ and for the situation when the HTR reactor is located close to the technological process, for the reactor outlet temperature equal to $1023 \mathrm{~K}$ and $1123 \mathrm{~K}$. The comparative calculations (Fig. 4) indicate that the pressure and temperature drops in $1 \mathrm{~km}$ long pipes does not lead to significant decrease in both efficiencies. Calculations carried out for three stage of the argon compressions (Fig. 5) and similar parameters as in the case presented in Fig. 3 show that three stage compression leads to decreasing of efficiencies on about one percent. Moreover, a reasonable increase of the reactor coolant pressure involves only a slight increase of both efficiencies (see Fig. 6). However, an increase of the outlet reactor temperature results in significant decrease of the efficiencies (see Fig. 3 vs. Fig. 2).

The results also reveal the importance of heat regeneration in the HP cycle. Diminishing temperature difference in the regenerative exchanger causes rapid increase in both efficiencies but also results in increasing heat transfer area. This temperature difference is constant in the whole exchanger, because argon is an ideal gas. Additionally, it can be noticed from the presented graphs for one compressing stage of the argon that increasing the temperature drop of the 'hot' argon in 'higher' heat exchanger causes rapid diminishing of both exergy and energy efficiencies. On the other hand, diminishing this temperature drop causes decrease of the heat flux delivered for higher temperature level technological process for assumed argon stream. This is one of the reasons for applying two or three stages of argon compression.

\section{Final remarks}

The results of the carried out analyses of the considered three cycle cogeneration systems with HTR reactor and intermediate $\mathrm{H}_{2} \mathrm{O}$ cycle used to transport energy from the reactor to technological system located, e.g., at a distance of $1 \mathrm{~km}$ from each other show that pressure and temperature drops of the energy carrier in pipes of the intermediate cycle does not eliminate these systems. The problem requires further investigations based on 
economical criteria and safety analyses.

The following other conclusions can be reported basing on the results of calculations:

- The energy and exergy efficiencies have small values despite applied cogeneration. It results mainly from low efficiency of the ClausiusRankine cycle.

- Temperature difference in the regenerative Ar-Ar heat exchanger and the argon temperature drop in the high temperature technological heat exchanger influence significantly the considered efficiencies. The decrease of these parameters involves significant increase of both efficiencies.

- Differences between efficiencies for the systems with one and three stages compression in the HP cycle are insignificant.

Acknowledgements This work was supported by the National Centre for Research and Development, Poland, under the strategic project Technologies Supporting Development of Safe Nuclear Power Engineering, task 1: 'Development of high temperature reactors for industrial purposes (HTRPL)', grant number $\mathrm{SP} / \mathrm{J} / 1 / 166183 / 12$.

Received 10 December 2014

\section{References}

[1] Mikielewicz D., Mikielewicz J.: Utilisation of bleed steam heat to increase the upper heat source temperature in low-temperature ORC. Arch. Thermodyn. 32(2011), 3, 57-71.

[2] Chmielniak T., Lepszy S., Czaja D.: The use of air-bottoming cycle as a heat source for the carbon dioxide capture installation of a coal-fired power unit. Arch. Thermodyn. 32(2011), 3, 89-103.

[3] Celiński Z., Strupczewski A.: Fundamentals of Nuclear Power, PWN, Warsaw 1984 (in Polish).

[4] Yildis B., Kazimi M.S.: Efficiency of hydrogen production systems using alternative nuclear energy technologies. Int. J. Hydrogen Energ. 31(2006), 77-92.

[5] Yanhui X., Huaiming J., Daxin Z.: HTGR Process Heat Application Study. Tsinghua Science and Technology 10/19(1996), 1, 40-44, ISSN 1007-2024.

[6] Kurh R.: HTR's role in process heat applications. Nucl. Eng. Des. 238(2008), 3013-3017. 
[7] Marmier A., FÜtTerer M.A.: Nuclear powered heat pumps for near-term process heat applications. Nuc. Eng. Des. 238(2008), 2272-2284.

[8] Hanuszkiewicz-Drapaea M., JęDrzejowski J.: Thermodynamic analysis of a cogeneration system. J. Power Technologies, papers.itc.pw.edu.pl, 2015.

[9] EBSILON Professional Documentation, https://www.steag-systemtechnologies.com

[10] Rietschel H., Reiss W.: Heiz und Luftungstechnik, 14th Edn. Springer Verlag, Berlin 1963.

[11] Szargut J., Morris D.R., Steward F.R.: Exergy Analysis of Thermal, Chemical, and Metallurgical Processes. Hemisphere Publishing Corporation, SpringerVerlag, Berlin 1988.

[12] Tani F., Haldi P. A., Favrat D.: Exergy-Based Comparison of the Nuclear Fuel Cycles of Light Water and Generation IV Rectors, http://moodle.epfl.ch/pluginfile.php/1092351/mod_resource/content/0/exergy_analysis_ucycle.pdf

[13] http://www.fchart.com/ees/

[14] List Reference Fluid Thermodynamic and Transport Properties Database (REFPROP), Ver. 9.1. National Institute of Standatrds and technology, Bouler 2013. 